Ann. Zootech., I976, 25 (4), 567-571.

NOTE

\title{
VARIATIONS DE LA MOTILITÉ ET DE LA FÉCONDANCE DES SPERMATOZOIDES DE BOUC
}

\author{
J.-M. CORTEEL
}

avec la collaboration technique de G. Barit, B. Lebtur, G. de MontgnY* et Y. Bentens:**

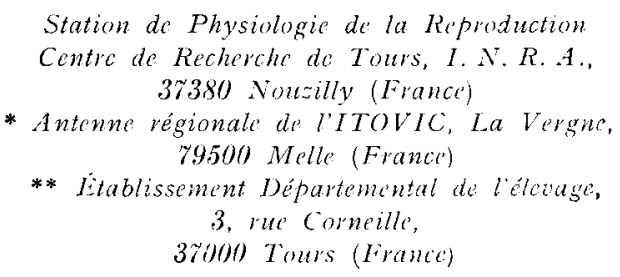

RÉSUMÉ

La motilité des spermatozoidles de bouc est fortement diminuée pendint trois mois de l'année après qu'ils aient été dilués et refroidis à $+4^{\circ} \mathrm{C}$. Ceci a été confirmé pour cleux boucs Alpins dont lo sperme a été récolté de juin à aon̂t. Conservé quelquess heures à $+t^{\circ}{ }^{\circ}(\mathrm{C}$, colui-ci a été utilisé pour inséminer 265 chèvres soumises à un traitement progestatif. Élevée en début et en fin d'essai (57,3 p. roo de M. B. ; 96 fenclles), la fertilité des chèvres inséminées est demeurés à un niveau très faible (I 5.4 P. I00; i69 femelles) penclant huit semaines consécutives. Pendant ces huit semaines, un autre groupe de I3,t chèrres soumisos au même traitement progestatil étaient inséminées avec le sperme des mêmes boucs mais récolté et congelé en périocle annuelle de motilité élevée : la fertilité cles chèvres inséminées a été cle $69,+1$ r. roo.

Dans notre expérience, la diminution de la motilité des spermatozoïdes de bouc dilués et refroidis à $+4^{\circ} \mathrm{C}$ est corrélée avec une diminution très sévìre de lenr fécondance. Cette diminution de motilité étant observée d'année cn année sur les mênues animaux, on peut pensel que la lécondance des spermatozoïles de boue climinue fortement quelques niois part an en saison non sexuelle.

\section{INTRODUCTION}

Chez le bouc, la motilité des spermatozoïdes après congélation et dégel évolue suivant le monnt où le sxeme a été récolté : olle est forte no it iminuée pendant trois mois au printemps ou ein été, selon les année:s (CORTEEI, I9jJ). Cette diminution 
de la motilité est également observée pendant les mêmes périodes après que le sperme ait été seulement dilué et refroidi à $4^{\circ} \mathrm{C}$. Dans le présent travail, nous avons cherché à savoir si cette baisse de la motilité des spermatozoïdes était associée à une baisse de leur fécondance en utilisant pendant l'été du sperme récolté à différentes saisons.

\section{MATÉRIEL E'T MÉTHODES}

\section{r. - Conditions de collecte et de conservation}

Le sperme a été récolté de deux boucs alpins âgés de 4 à 5 ans, à raison de deux éjaculats par semaine. Dès la récolte, les éjaculats ont été dilués à la température ambiante dans une solution de Krebs-Ringer-Phosphate contenant $0,05 \mathrm{M}$ de glucose et centrifugés ( $15 \mathrm{mn}$ à $600 \mathrm{~g}$ ).

a) Sperme vécolté de juin à acùt, conservé à $+4^{\circ} \mathrm{C}$.

$A$ près élimination de la solution de lavage, Jes spermatozoïdes ont été resuspendus dans un milieu à base de lait de vache écrémé contenant o, $05 \mathrm{M}$ de glucose à une concentration de $0,5 \times$ Io $^{9}$ spermatozoïles par $\mathrm{ml}$. Après refroidissement ì $+4^{\circ} \mathrm{C}$, la semence a été répartie clans des paillettes de $0,5 \mathrm{ml}$; pour chaque éjaculat, le contenu de deux paillettes a été réchauffé à $+37^{\circ} \mathrm{C}$, dilué Io fois et examiné au microscope à contraste de phase pour estimation du pourcentage de spermatozoïles mobiles et de leur motilité.

b) Sperme récolté de décembre à juin, conservé à $-196^{\circ} \mathrm{C}$.

$\Lambda$ près élimination de la solution de lavage, les spcrmatozoüdes ont été prédilués dans le même milieu que précédemment, puis refroidis à $+f^{\circ} \mathrm{C}$. A cette température, la concentration de la semence $\left(\mathrm{r} \times \mathrm{IO}^{9} \mathrm{spz} / \mathrm{ml}\right)$ a été réduite de moitié par l'adjonction du volume approprié du même dilueur contenant $14 \mathrm{p}$. Ioo de glycérol. Répartie dans des paillettes de $0,2 \mathrm{ml}$ ou de $0,5 \mathrm{ml}$, la semence a été congclée trois heures plus tard dans les vapeurs d'azote et conservée à - $196^{\circ} \mathrm{C}$.

Deux à trois jours après la congélation, le contenu de deux paillettes de chaque éjaculat a été dégelé à $+37^{\circ} \mathrm{C}$ puis dilué ıo fois dans le dilueur de congélation pour être examiné au microscope à contraste de phase. Le pourcentage de spermatozoïdes réanimés et leur motilité ont été estimés par un observateur expérimenté. La motilité individuelle des spermatozoïles a été notée selon une échelle de valeurs croissantes de o à 5 .

\section{2. - L'épréuve de fécondance (I 2 juin-28 août)}

Le sperme a été utilisé pour l'insémination de 399 chèvres dont l'œstrus et l'ovulation ont été provoqués par un traitement progestatif avant le début de la saison sexuelle (CorTEEL, 1975). Toutes les chèvres ont été inséminécs dans l'endocervix une première fois $32 \pm 2$ heures après la cessation du traitement progestatif, puis une seconde fois I \& à 24 heures plus tard. Le nombre de spermatozoïdes mis en place au cours de l'œestrus induit a varié de zoo à 500 millions pour I 34 chèvres inséminées avec le sperme congelé ; il a toujours été de 500 millions pour les 265 chèvres inséminées avec le spcrme conservé à $+4^{\circ} \mathrm{C}$. Les résultats de fertilité sont exprimés en pour cent de mises bas. Ils indiquent la fécondance des spermatozoïdes inséminés.

\section{RÉSULTATS}

\section{I. - La qualité du sperme}

a) Sperme conservé à $+4^{\circ} \mathrm{C}:$ la motilité des spermatozoïdes après dilution et refroidissement a varié de 3,3 à 4,5 selon les éjaculats mais ceux-ci contenaient toujours au moins 69 p. Ioo de spermatozoïdes fléchants. Ils ont tous été retenus pour l'épreuve de fécondance. L'évaluation de la motilité des spermatozoïdes des éjaculats utilisés est indiquée à la figure I. 


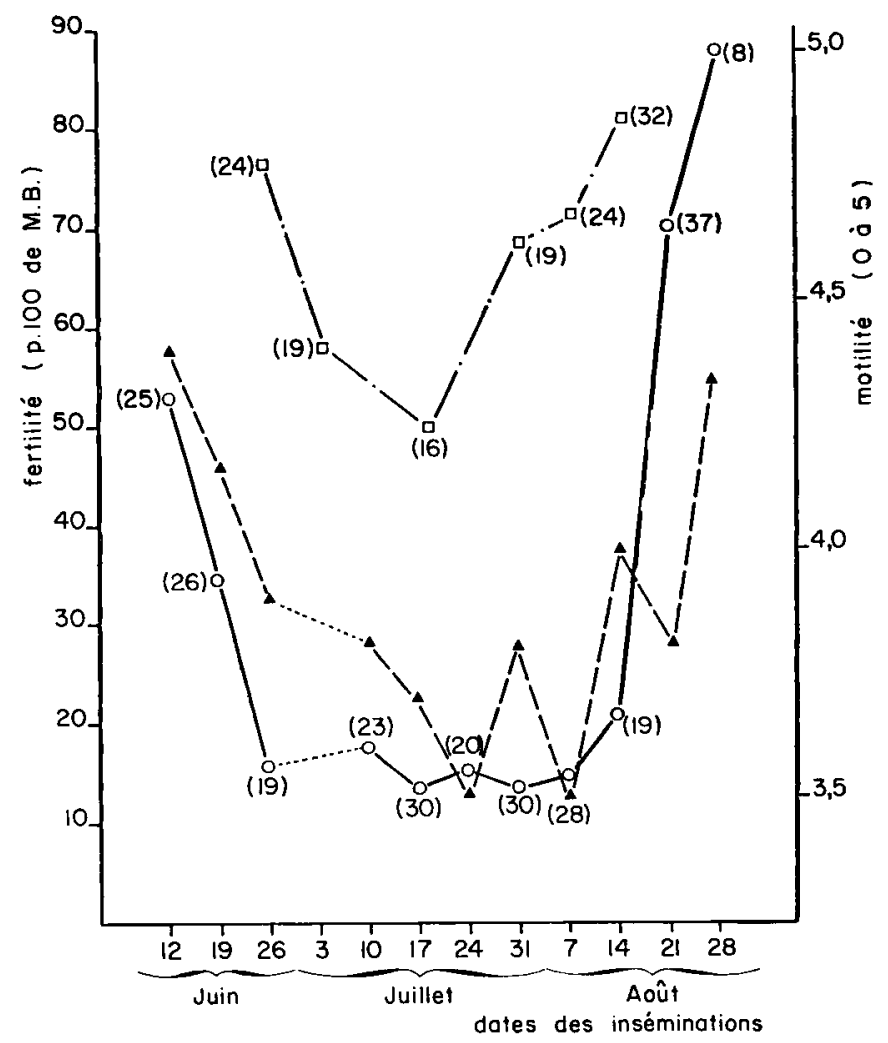

Fic. I. - T'ariations de la ficondance et de la motilité des spermatozoides de Bouc all cours de l'annie

$(\quad)=$ Nombre de femelles inséninces

o- - Fertilité des spermatozoides conservés ì $+4^{\circ \mathrm{CC}}$

a- - Motilité des spermatozoides conservés à !- $4^{\circ} \mathrm{C}$

[ - - - lertilité des spermatozoides récoltés de décenbre a juin et conservós a - Igr $0^{\circ} \mathrm{C}$

b) Sperme congelé: la motilité des spermatozoïdes après dilution et refroidissement à $+4^{\circ} \mathrm{C}$ a été d'un niveau élevé et constant (au moins égale à 4). Après congélation, 60 p. roo des éjaculats présentaient plus de 30 p. roo de spermatozoïdes mobiles animés d'une motilité individuelle au moins égale à 3 . Ces éjaculats ont été conservés pour la mesure ultérieure de leur fécondance.

\section{2. - Fécondance du sperme}

Dans la seconde quinzaine de juin, la fertilité des spermatozoïdes conservés à $4^{\circ} \mathrm{C}$ passe de $5^{2} \mathrm{p}$. IoO à $\mathbf{I} \mathbf{5}$ p. IOO. Fille demeure à ce très faible niveau jusqu'à la mi-août, moment à partir duquel, elle s'élève au-delà de 60 p. Ioo. La fécondance du sperme récolté et inséminé au cours des deux premières et des deux dernières semaines de 1'essai est significativement supérieure à celle du sperme récolté et inséminé du 26 juin au $I_{4}$ août $(57,3$ p. Ioo contre 15,4 p. IOO ; P $<0,01)$.

La diminution de la fécondance et son retour à un niveau élevé précédent d'une 
semaine environ la diminution puis le retour à un niveau élevé de la motilité individuelle des spermatozoïdes. La fécondance et la motilité sont étroitement liées ( $r$ : 0,$88 ; \mathrm{P}<\mathrm{o,OI}$; fig. I).

Le sperme congelé a été utilisé du 26 juin au I4 août, période où la fertilité du sperme conservé à $+4^{\circ} \mathrm{C}$ était à son niveau le plus bas. La fécondance du sperme congelé est alors très significativement supérieure à celle du sperme conservé à $+4^{\circ} \mathrm{C}: 69,4$ p. Ioo contre $5_{5,4}$ p. Ioo. En dépit des apparences, elle ne diffère pas significativement d'une semaine sur l'autre, même dans les cas extrêmes (fig. I).

\section{DISCUSSION}

La diminution de la motilité des spermatozö̈des observée dans la présente expérience en été, avait été constatée antérieurement au printemps ou en été sans que nous ayons pu relier ces variations à l'âge des animaux ( ${ }^{r e}$ saison non sexuelle de la vie de l'animal, ou saisons non sexuelles ultérieures). Cette diminution demeure inexpliquée mais elle suit immédiatement la dininution de la fécondance des spermatozoïdes. La baisse de fertilité ne peut être due à un nombre insuffisant de spermatozoïdes mobiles par I.A. comme nous l'avons observé ailleurs (CORTEEL, I975), car toutes les chèvres inséminées avec du sperme conservé à $4^{\circ} \mathrm{C}$ ont reçu un nombre élevé et constant de spermatozoïdes mobiles.

Le haut niveau de fertilité obtenu entre le 26 juin et le I4 août avec le sperme congelé indique que la femelle n'est pas la cause de la moindre fertilité obtenue à cette époque avec le sperme conservé à $-^{-} 4^{\circ} \mathrm{C}$. Toutes les femelles étaient dans les mêmes conditions physiologiques de lactation.

\section{CONCLUSION}

Ainsi, notre expérience a démontré qu'une très basse fertilité, observée au milieu de l'été, était liée principalement à la qualité du sperme produit pendant cette même période, non à une chute de fertilité des femelles.

La période de faible motilité des spermatozoïdes a été contemporaine de la période de basse fertilité, quoiqu'elle s'est prolongée pendant une semaine où la fertilité était déjà élevée. La diminution de la motilité des spermatozoïdes observée chaque année chez les mêmes animaux depuis le début de leur vie sexuelle, suggère que la fécondance des spermatozoïdes de bouc diminue fortement quelques mois par an en saison non sexuelle. 


\title{
SUMMARY
}

\author{
SEASONAL, VARIATIONS IN GOAT SPERMATOZOA MOTILITY \\ AND FERTILIZING CAPACITV
}

The motility of goat spermatozoa is drastically reduced during a three month period within the non breeding season after the semen has been diluted and cooled to $4^{\circ} \mathrm{C}$. Ejaculates from 2 billy-goats of the Alpine breed were collected regularly and diluted and cooled from June through August during which time sperm motility declined, remained at a low level and then increased back to its initial value. Spermatozoa were stored for a few hours only at $4^{\circ} \mathrm{C}$ and inseminated into 265 progestagen treated goats. The fertility of the inseminated goats was high at the beginning and end of the period $(57,3 \mathrm{p}$. Ioo of 96 inseminated goats did kid), but fell to a very low level during 8 consecutive weeks ( 15,4 p. Ioo for I 68 goats). During these 8 weeks another group of 134 goats were inseminated in the same conditions of hormonal treatment, using ejaculates from the same billy-goats, but collected and deep frozen during annual periods of high motility. Under these conditions, 69,4 p. Ioo of the goats kidded.

The results suggest that the periodical decrease in motility of diluted and cooled goat spermatozoa is related to a severe decrease in their fertilizing ability. Since this decrease in motility has been observed repeatedly in the same animals, it is possible that goat sperm loose most of their fertilizing power during two or three months each year.

\section{RÉFÉRENCES BIBLIOGRAPHIQUES}

Cortael J.-M., I975. La production du sperne chez le Bouc : variations saisonnières de la quantité et de la qualité du sperme récolté selon l'âge des animaux. Comples rendus des Journées de la Recherche Ovine et Caprine. Tome $\mathrm{r}$, Espèce Caprine, 4-I7.

Contris J.-M., I975. Le contrôle du cycle sexuel de la Chèvre. Comptes rendus des Journées de la Recherche Ovine et Caprine, tome I, Espèce Caprine, 28-47. 